\title{
Ação do colírio de fenilefrina $10 \%$ no posicionamento palpebral de indivíduos normais
}

\author{
Phenylephrine 10\% eye drop action in the eyelid position in healthy subjects
}

Tânia Pereira Nunes ${ }^{1}$

Suzana Matayoshi ${ }^{2}$
Trabalho realizado no Setor de Plástica Ocular da Clínica Oftalmológica do Hospital das Clínicas da USP - São Paulo (SP) - Brasil.

${ }^{1}$ Médica pós-graduanda da Clínica Oftalmológica do Hospital das Clínicas da Universidade de São Paulo USP - São Paulo (SP) - Brasil.

${ }^{2}$ Chefe do Setor de Plástica Ocular da Clínica Oftalmológica do Hospital das Clínicas da USP - São Paulo (SP) - Brasil.

Endereço para correspondência: Tânia Pereira Nunes. Rua Rio Grande do Sul, 170 - Apto. 204 - Teixeira de Freitas (BA) CEP 45996-026

E-mail: nunestp@hotmail.com

Recebido para publicação em 06.03.2008

Última versão recebida em 11.04.2008

Aprovação em 16.05.2008

\begin{tabular}{|l|}
\hline RESUMO \\
\hline Objetivos: Determinar a ação de uma gota do colírio de fenilefrina $10 \%$ \\
sobre as pálpebras superior e inferior de indivíduos normais; verificar a \\
ocorrência de alterações no posicionamento das pálpebras superior e \\
inferior do olho contralateral, após instilação desta medicação. Métodos: \\
Estudo prospectivo observacional, do qual participaram 24 indivíduos \\
normais, com idade entre 20 e 39 anos, submetidos à instilação de uma \\
gota do colírio de fenilefrina $10 \%$ no fundo de saco conjuntival superior, \\
aleatoriamente em um dos olhos. Todos os indivíduos foram filmados \\
antes e após a instilação do colírio (3, 10, 15, 30, 45 e 60 minutos). As \\
imagens foram submetidas ao processamento digital e editadas para \\
análise das medidas palpebrais. Foi traçada uma linha horizontal do canto \\
medial até o canto externo. Considerou-se a altura da pálpebra superior \\
a distância entre o ponto mais alto da margem palpebral superior e a linha \\
horizontal traçada. A altura palpebral inferior foi avaliada como a distân- \\
cia entre o ponto mais baixo da margem palpebral inferior e a referida \\
linha. Resultados: Em relação à pálpebra superior, a medida antes da \\
instilação da fenilefrina $10 \%$ diferiu significativamente dos demais \\
momentos (p<0,001) com elevação média da pálpebra superior de \\
0,79 mm e retração de 0,37 mm na altura palpebral inferior. Em relação \\
ao olho contralateral, observou-se queda da pálpebra superior em prati- \\
camente todos os momentos estudados, com o menor nível aos 3 minutos \\
(queda média de 0,68 mm). A pálpebra inferior contralateral mostrou \\
elevação média de 0,37 mm. Conclusões: A instilação de uma gota do \\
colírio de fenilefrina $10 \%$ em olhos de indivíduos normais altera a \\
posição da pálpebra superior do olho testado, assim como a da pálpebra \\
inferior. O posicionamento da pálpebra superior e inferior do olho \\
contralateral também se alteram.
\end{tabular}

Descritores: Pálpebras/fisiologia; Pupila; Fenilefrina/administração \& dosagem; Fenilefrina/ farmacologia; Soluções oftálmicas

\section{INTRODUÇÃO}

Existem poucos estudos na literatura sobre o efeito de drogas no posicionamento vertical das pálpebras. Este assunto é importante na análise da dinâmica palpebral, principalmente em afecções como a blefaroptose (BP).

$\mathrm{O}$ colírio de fenilefrina $0,125 \%$ tem sido utilizado na avaliação da ptose palpebral (PP) por denervação simpática (síndrome de Horner) ${ }^{(1)}$. Como é um agonista simpatomimético, sua aplicação estimula a contração das fibras lisas do músculo de Müller (MM). Considera-se o teste positivo quando há aumento da fenda palpebral 10 minutos após a instilação do colírio na pálpebra ptótica, sendo o teste feito com o colírio na concentra- 
ção de $10 \%$, considerado importante na avaliação pré-operatória de indivíduos com PP para programação subseqüente da Müllerectomia $^{(2)}$. Entretanto, são poucos os estudos que avaliam a resposta das pálpebras normais diante da instilação do colírio de fenilefrina ${ }^{(3-4)}$.

O presente estudo foi desenvolvido, tendo como sujeitos indivíduos normais, com os objetivos de:

- determinar o efeito do colírio de fenilefrina $10 \%$ nas pálpebras superior e inferior;

- verificar a ocorrência de alterações no posicionamento das pálpebras superior e inferior do olho contralateral, após instilação do colírio.

\section{MÉTODOS}

Estudo prospectivo observacional, aprovado pelo comitê de ética da Instituição. Foram selecionados 24 voluntários, com idade entre 20 e 39 anos. Os critérios de exclusão foram: cirurgia ocular e/ou palpebral prévias, ptose ou retração palpebral, exoftalmia, enoftalmia, ametropias com equivalente esférico maior que 5 dioptrias, visão corrigida menor que 20/20 nos dois olhos, hipertensão arterial sistêmica e doença cardiovascular. Foi instilada uma gota do colírio de fenilefrina $10 \%$ no fundo de saco conjuntival superior. A aplicação do colírio foi feita de forma aleatória, em um dos olhos.

Todos os indivíduos foram filmados antes e após a instilação do colírio de fenilefrina $10 \%$, utilizando para captação da imagem uma câmera de vídeo digital, Sony DCRHC40, à distância de $1 \mathrm{~m}$, com o paciente sentado e sua cabeça posicionada em um aparato para mento e testa. Foi fixada uma régua próxima da região frontal do paciente, no sentido horizontal, possibilitando assim padronizar as medidas de todas as imagens. O indivíduo foi orientado a permanecer em posição primária do olhar e piscando normalmente durante a filmagem. A documentação do vídeo foi feita imediatamente antes da instilação e depois de 3, 10, 15, 30, 45 e 60 minutos.

Foram utilizados os programas Pinnacle Studio Version (versão 9.0) para edição dos vídeos e o Image J 1.34n para análise das medidas palpebrais.

Foi traçada uma linha horizontal, tomando-se por referência o canto medial e o canto lateral. $\mathrm{O}$ efeito da fenilefrina $10 \%$ sobre a pálpebra superior (PS) foi analisado pela distância entre o ponto mais alto da margem palpebral superior e a linha horizontal (M1) e, sobre a pálpebra inferior (PI), avaliando-se a distância entre o ponto mais baixo da margem palpebral inferior e a linha horizontal (M2) (Figura1).

Os dados obtidos foram submetidos à avaliação descritiva. Para as variáveis quantitativas, a análise estatística foi feita observando-se os valores mínimos, máximos, além do cálculo de médias e desvios-padrão e medianas. Para averiguar o comportamento do grupo considerando as condições estudadas, fez-se uso da técnica Análise de Variância com medidas repetidas. O nível de significância adotado foi de $5 \%$.

\section{RESULTADOS}

Em relação à PS, a M1 antes da instilação da fenilefrina $10 \%$ diferiu significativamente dos demais momentos $(\mathrm{p}<0,001)$ com aumento da distância entre a pálpebra superior e a linha traçada (M1), ou seja, houve retração da pálpebra superior até a medida feita após 30 minutos da instilaçào do colírio (Tabela 1). Na PI, esta diferença foi significativa $(p<0,001)$ até 15 minutos após a instilação, ocorrendo aumento da distância entre a pálpebra inferior e a linha traçada (M2), ou seja, houve retração da pálpebra inferior (Tabela 2).

$\mathrm{O}$ pico de aumento de M1 e de M2 ocorreu após 10 minutos da instilação do colírio. O aumento médio de M1 foi de $0,79 \mathrm{~mm}$. O aumento médio de M2 foi de $0,37 \mathrm{~mm}$. Somandose a alteração ocorrida na PS com a da PI, houve um aumento médio de $1,16 \mathrm{~mm}$ na fenda palpebral vertical.

Em relação ao olho contralateral, observou-se diminuição do M1 em praticamente todos os momentos estudados, não havendo significância estatística somente aos 30 minutos. A diminuição mais acentuada ocorreu três minutos após a instilação da fenilefrina $10 \%$, (Tabela 3), com queda média de $0,68 \mathrm{~mm}$.

Em relação à PI do olho contralateral, observou-se a diminuição do M2 de forma semelhante, também com pico aos três minutos, com diminuição média de $0,37 \mathrm{~mm}$ (Tabela 4). $\mathrm{O}$ único momento em que a diferença não foi significativa, quando comparado com a medida inicial, ocorreu também aos 30 minutos.

No olho contralateral, as alterações da PS e PI, aos três minutos, diminuíram a fenda palpebral em cerca de $1,05 \mathrm{~mm}$.

O gráfico 1 mostra a comparação do posicionamento palpebral superior no olho testado e no contralateral, sendo possível observar o aumento do M1 no olho testado e a diminuição do M1 no olho contralateral.

O gráfico 2 mostra a comparação do posicionamento da PI no olho testado e no olho contralateral, podendo-se observar a retração palpebral inferior do olho testado e a diminuição do M2 no olho contralateral.

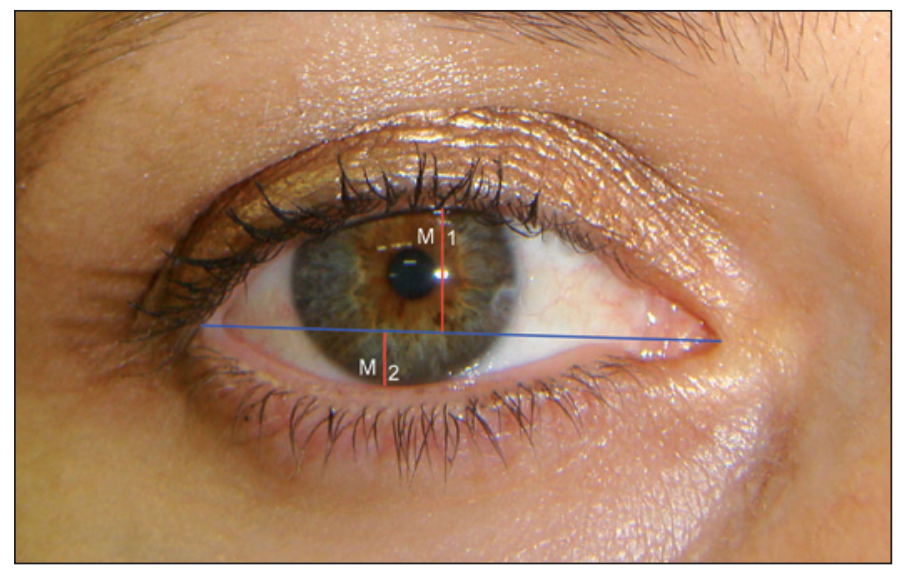

Figura 1 - M1= distância entre o ponto mais alto da margem palpebral superior e a linha horizontal e M2= distância entre o ponto mais baixo da margem palpebral inferior e a linha horizontal 
Tabela 1. Valores da média, desvio-padrão, mediana, valor mínimo e valor máximo da distância entre o ponto mais alto da pálpebra superior e a linha que une o canto externo ao interno, em milímetros, nos momentos de avaliação, após a instilação de colírio de fenilefrina $10 \%$

\begin{tabular}{|c|c|c|c|c|c|c|c|}
\hline Momento & $\mathrm{n}$ & Média & Desvio-padrão & Mediana & Mínimo & Máximo & $p^{*}$ \\
\hline Pré & 24 & 5,88 & 0,84 & 5,90 & 3,79 & 7,24 & \\
\hline $3 \min$ & 24 & 6,38 & 0,85 & 6,48 & 4,00 & 8,33 & $<0,001$ \\
\hline $10 \mathrm{~min}$ & 24 & 6,67 & 0,98 & 6,77 & 4,06 & 9,00 & $<0,001$ \\
\hline $15 \mathrm{~min}$ & 24 & 6,41 & 1,00 & 6,42 & 3,87 & 8,67 & $<0,001$ \\
\hline $30 \mathrm{~min}$ & 24 & 6,22 & 0,91 & 6,29 & 3,82 & 8,39 & $<0,001$ \\
\hline $45 \mathrm{~min}$ & 24 & 5,91 & 0,93 & 5,96 & 3,60 & 7,81 & 0,65 \\
\hline $60 \mathrm{~min}$ & 24 & 5,87 & 0,96 & 5,81 & 3,58 & 7,81 & 1,0 \\
\hline
\end{tabular}

Tabela 2. Valores da média, desvio-padrão, mediana, valor mínimo e valor máximo da distância entre o ponto mais baixo da margem palpebral inferior e a linha horizontal no olho testado, em milímetros, nos vários momentos do exame após a instilação de colírio de fenilefrina $10 \%$

\begin{tabular}{|c|c|c|c|c|c|c|c|}
\hline Momento & $\mathbf{n}$ & Média & Desvio-padrão & Mediana & Mínimo & Máximo & $\mathbf{p}^{*}$ \\
\hline Pré & 24 & 3,12 & 0,62 & 3,11 & 2,12 & 4,29 & \\
\hline $10 \mathrm{~min}$ & 24 & 3,49 & 0,62 & 3,29 & 2,35 & 4,60 & $<0,001$ \\
\hline $30 \mathrm{~min}$ & 24 & 3,19 & 0,70 & 3,17 & 2,00 & 4,55 & 0,367 \\
\hline $45 \mathrm{~min}$ & 24 & 3,12 & 0,58 & 3,00 & 2,33 & 4,40 & 0,940 \\
\hline $60 \mathrm{~min}$ & 24 & 3,03 & 0,55 & 2,92 & 2,15 & 4,05 & 0,074 \\
\hline
\end{tabular}

Tabela 3. Valores da média, desvio-padrão, mediana, valor mínimo e valor máximo da distância entre o ponto mais alto da margem palpebral superior e a linha horizontal no olho contralateral, em milímetros, nos momentos de avaliação

\begin{tabular}{|c|c|c|c|c|c|c|c|}
\hline Momento & $\mathbf{n}$ & Média & Desvio-padrão & Mediana & Mínimo & Máximo & $p^{*}$ \\
\hline Pré & 24 & 5,93 & 0,99 & 5,78 & 3,67 & 7,81 & \\
\hline $3 \mathrm{~min}$ & 24 & 5,25 & 0,94 & 5,08 & 3,23 & 7,33 & $<0,001$ \\
\hline $10 \mathrm{~min}$ & 24 & 5,46 & 0,90 & 5,46 & 3,45 & 7,00 & $<0,001$ \\
\hline $15 \mathrm{~min}$ & 24 & 5,56 & 0,87 & 5,48 & 3,33 & 6,91 & $<0,001$ \\
\hline $30 \mathrm{~min}$ & 24 & 5,69 & 0,82 & 5,68 & 3,33 & 6,85 & 0,014 \\
\hline $45 \min$ & 24 & 5,58 & 0,81 & 5,54 & 3,67 & 6,88 & $<0,001$ \\
\hline $60 \mathrm{~min}$ & 24 & 5,64 & 0,88 & 5,72 & 3,67 & 7,19 & $<0,001$ \\
\hline
\end{tabular}

\begin{tabular}{|c|c|c|c|c|c|c|c|}
\hline Momento & $\mathbf{n}$ & Média & Desvio-padrão & Mediana & Mínimo & Máximo & $p^{*}$ \\
\hline Pré & 24 & 3,06 & 0,76 & 2,94 & 1,69 & 5,48 & \\
\hline $3 \min$ & 24 & 2,69 & 0,61 & 2,59 & 1,55 & 4,52 & $<0,001$ \\
\hline $10 \min$ & 24 & 2,73 & 0,66 & 2,56 & 1,67 & 4,76 & $<0,001$ \\
\hline $15 \min$ & 24 & 2,88 & 0,62 & 2,88 & 1,67 & 4,70 & $<0,001$ \\
\hline $30 \mathrm{~min}$ & 24 & 2,95 & 0,65 & 2,86 & 1,64 & 4,75 & 0,059 \\
\hline $45 \mathrm{~min}$ & 24 & 2,91 & 0,65 & 2,81 & 1,94 & 5,08 & 0,004 \\
\hline $60 \min$ & 24 & 2,91 & 0,71 & 2,81 & 1,76 & 5,30 & 0,016 \\
\hline
\end{tabular}

\section{DISCUSSÃO}

O teste da instilação do colírio de fenilefrina $10 \%$ faz parte da semiologia das BP. A elevação palpebral após a instilação da medicação é interpretada como teste positivo, sendo deter- minante para a indicação da técnica de Müllerectomia para o tratamento cirúrgico da $\mathrm{PP}^{(2)}$.

Existe controvérsia quanto à melhor dosagem $(2,5 \%$ ou $10 \%$ ), sendo relatados efeitos sistêmicos indesejáveis como crise hipertensiva e alterações cardiovasculares ${ }^{(5)}$. Estudo recente, 


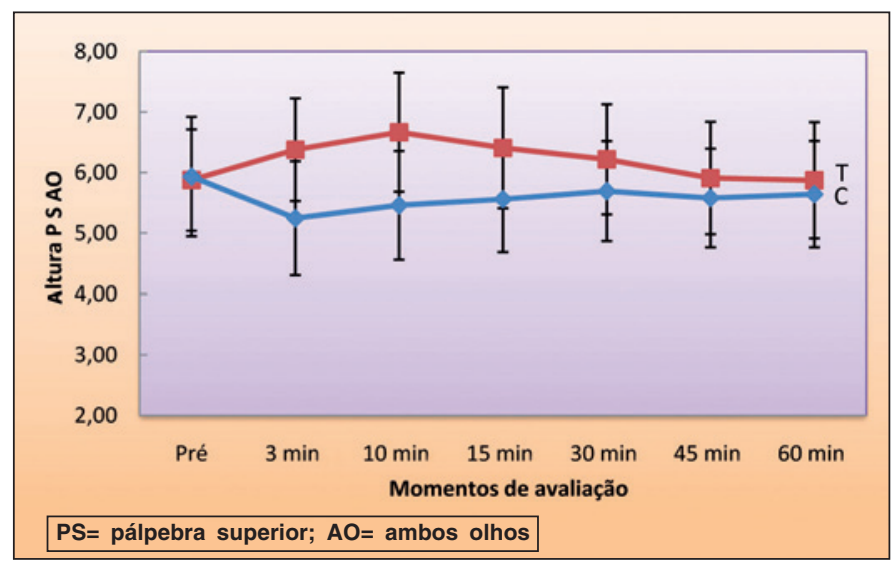

Gráfico 1 - Efeito da instilação de colírio de fenilefrina $10 \%$ na altura palpebral superior do olho testado (T) e no contralateral (C).

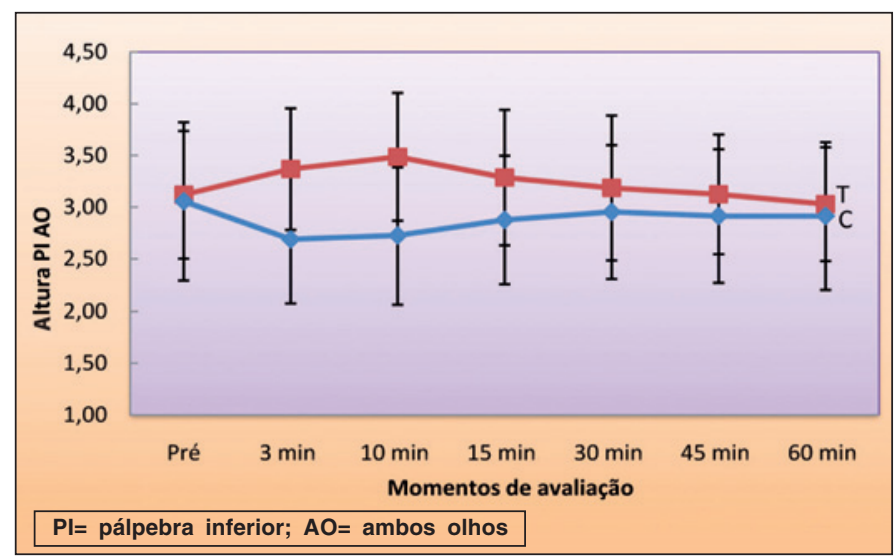

Gráfico 2 - Efeito da instilação de colírio de fenilefrina $10 \%$ na altura palpebral inferior do olho testado (T) e do olho contralateral (C)

entretanto, demonstrou a segurança do uso do colírio a $10 \%{ }^{(6)}$. A resposta da elevação da pálpebra na concentração de $10 \%$ parece ser melhor que a de $2,5 \%$, em indivíduos normais ${ }^{(4)}$.

A maioria dos autores emprega de 2 a 4 gotas de colírio de fenilefrina nos testes, diferentemente do presente estudo, onde houve resposta, mesmo com somente uma gota do colírio ${ }^{(2,7)}$. A elevação palpebral foi menor que o encontrado na literatura, provavelmente, devido a esse fator $(0,79 \mathrm{~mm}$ contra $1,3 \mathrm{~mm}$ a $1,6 \mathrm{~mm})^{(3-4)}$.

A elevação palpebral foi máxima 10 minutos após a instilação do colírio, sendo decorrente da estimulação direta da fenilefrina sobre o $\mathrm{MM}^{(3)}$. O presente estudo demonstrou que o colírio age também sobre o músculo tarsal inferior, levando à retração da PI em cerca de $0,37 \mathrm{~mm}$.

Em relação ao olho contralateral, foram identificadas modificações no posicionamento palpebral: a pálpebra superior abaixou $(0,68 \mathrm{~mm})$ e a pálpebra inferior subiu $(0,37 \mathrm{~mm})$, logo nos primeiros minutos, com retorno rápido à posição inicial. Esta observação sugere um interessante mecanismo de balanceamento e relação entre as pálpebras do lado direito e esquerdo, no tocante à abertura palpebral.
Os dois únicos estudos existentes sobre este assunto, analisam uma casuística pequena ${ }^{(3-4)}$. Ainda, as pálpebras contralaterais foram utilizadas como controles, ignorando-se a possibilidade de alterações secundárias, desencadeadas pela medicação, como foi demonstrado no presente estudo, quando ficou claramente demonstrado que a aplicação de uma gota de fenilefrina $10 \%$ em um dos olhos, aumenta a fenda palpebral vertical em 1,16 mm e desencadeia diminuição da medida de $1,05 \mathrm{~mm}$ na fenda palpebral contralateral.

Aceita-se, atualmente, que o controle de ambos músculos levantadores da pálpebra superior (MLPS) seja feito por um único núcleo oculomotor (núcleo central caudal) ${ }^{(8)}$, sendo que esse fato explica a ocorrência de ptose contralateral após cirurgia de correção de ptose unilateral (diminuição da estimulação enviada aos MLPS de ambos os lados: aplicação da Lei de Hering para os MLPS).

No caso do teste da fenilefrina, não seria apropriada a aplicação da Lei de Hering, pois a medicação age no MM, cuja via nervosa não é a mesma do MLPS. A explicação mais plausível estaria relacionada à ação dos mecanoreceptores no MM que influenciaria a contração tônica do MLPS. ${ }^{(9)}$ Esses mecanoreceptores, sensíveis ao estiramento, evocam estímulo aferente ao núcleo mesencefálico trigeminal que, por sua vez, estimulariam o núcleo central caudal do oculomotor, provocando contração tônica involuntária do MLPS bilateralmente ${ }^{(9)}$. A contração do MM por mecanismo inverso poderia, então, diminuir a aferência para o núcleo, levando, então, ao abaixamento de ambas pálpebras superiores, só que do lado da medicação, em virtude da ação da droga, não haveria abaixamento.

Em relação à PI contralateral, a presença de mecanoreceptores atuando no músculo tarsal inferior poderia, de maneira semelhante, explicar o comportamento do posicionamento palpebral, embora isso ainda não esteja demonstrado.

Vale a pena ressaltar que as alterações palpebrais contralaterais só puderam ser registradas devido ao processamento digital da imagem e maior sensibilidade das mensurações computadorizadas, que possibilitaram a detecção de diferenças pequenas, muitas vezes não perceptíveis ao exame ectoscópico.

\section{CONCLUSÕES}

A instilação de uma gota do colírio de fenilefrina $10 \%$ em olhos de indivíduos normais altera a posição da pálpebra superior do olho testado, assim como da pálpebra inferior. Ocorre também alteração do posicionamento da pálpebra superior e inferior do olho contralateral.

\section{ABSTRACT}

Purpose: To determine the upper and lower eyelid position with a single drop of $10 \%$ phenylephrine in normal indivi- 
duals and observe positional lid changes in upper and lower lids of the opposite eye. Methods: Prospective observational study including 24 healthy subjects of both genders (age range: 20-39 yrs) submitted to instillation of a single drop of $10 \%$ phenylephrine in the upper fornix of one randomly chosen eye. A video camera recorded the images of both eyes before and after eye drop application at 3, 10, 15, 30, 45 and 60 minutes. The images were digitally processed and edited to analyze lid measurements (upper lid height: distance between the highest upper margin lid point and a horizontal line traced from the medial to the lateral canthus; lower lid height: distance between the lowest margin lid point and the above-mentioned line). Results: Before and after drug instillation, upper lid height was significantly different $(p<0.001)$ at all moments with a mean elevation of $0.79 \mathrm{~mm}$. The lower lid height changed too with a mean retraction of $0.37 \mathrm{~mm}$. In the fellow eye upper lid drop was observed at almost all moments with the lowest value at 3 minutes (mean reduction of $0.68 \mathrm{~mm})$ and elevation $(0.37 \mathrm{~mm})$ of lower lid. Conclusions: This experiment showed that a single drop of $10 \%$ phenylephrine acted not only on the upper eyelid but also on lower lid of the tested eye. Control mechanism of lid opening and closing changed upper and lower lid positioning of the opposite eye in healthy subjects.
Keywords: Eyelids/physiology;Pupil; Phenylephrine/administration \& dosage; Phenylephrine/pharmacology; Ophthalmic solutions

\section{REFERÊNCIAS}

1. Glatt HJ, Putterman AM, Fett DR. Müller's muscle-conjunctival resection procedure in the treatment of ptosis in Horner's syndrome. Ophthalmic Surg. 1990;21(2):93-6.

2. Putterman AM, Fett DR. Müller's muscle in the treatment of upper eyelid ptosis: a ten-year study. Ophthalmic Surg. 1986;17(6):354-60.

3. Munden PM, Kardon RH, Denison CE, Carter KD. Palpebral fissure responses to topical adrenergic drugs. Am J Ophthalmol. 1991;111(6):70610.

4. Felt DP, Frueh BR. A pharmacologic study of the sympathetic eyelid tarsal muscles. Ophthal Plast Reconstr Surg. 1988;4(1):15-24.

5. Fraunfelder FT, Scafidi AF. Possible adverse effects from topical ocular $10 \%$ phenylephrine. Am J Ophthalmol. 1978;85(4):447-53.

6. Diniz Filho A, Frasson M, Mérula RV, Morais PR, Cronemberger S. Cardiovascular and pupillary effects of topical ophthalmic 2.5 and $10.0 \%$ phenylephrine in healthy volunteers. Arq Bras Oftalmol. 2007;70(6):961-6.

7. Ben Simon GJ, Lee S, Schwarcz RM, McCann JD, Goldberg RA. Muller's muscle-conjunctival resection for correction of upper eyelid ptosis: relationship between phenylephrine testing and the amount of tissue resected with final eyelid position. Arch Facial Plast Surg. 2007;9(6):413-7.

8. Schmidtke K, Büttner-Ennever JA. Nervous control of eyelid function. A review of clinical, experimental and pathological data. Brain. 1992;115(1): 227-47.

9. Matsuo K. Stretching of the Mueller muscle results in involuntary contraction of the levator muscle. Ophthal Plast Reconstr Surg. 2002;18(1):5-10.
Ao enviar um artigo para publicação,

leia ATENTAMENTE as instruções para autores,

constante no final de cada fascículo. 